\title{
Transmission line printed using silver nanoparticle ink on FR-4 and polyimide substrates
}

\author{
Sung-min Sim ${ }^{1}$, Yeonsu Lee ${ }^{1}$, Hye-lim Kang ${ }^{1}$, Kwon-Yong Shin², Sang-Ho Lee ${ }^{2}$ and Jung-Mu Kim ${ }^{3 *}$
}

\begin{abstract}
In this paper, nano-silver ink-jet printed transmission lines were fabricated to investigate RF performance on both flame retardant (FR-4) and polyimide (PI) as rigid and flexible substrates. The transmission lines were printed by using the ink-jet printer with velocity of $3.5 \mathrm{~mm} / \mathrm{s}$ and were sintered in a convection oven with $250^{\circ} \mathrm{C}$. The RF performance of transmission lines was simulated and measured at low frequencies. The transmission loss is measured to be $0.22 \mathrm{~dB} @ 1 \mathrm{GHz}$ and $0.32 \mathrm{~dB} @ 1 \mathrm{GHz}$, respectively, for the FR-4 and PI substrates, respectively. The return loss has over $16 \mathrm{~dB}$ for FR-4 substrate and over $12 \mathrm{~dB}$ for PI substrate. The RF performance of transmission line was investigated and discussed in regard to an influence by two substrates. The measured RF performance of fabricated transmission lines results in the possibility that flexible device is explored in low frequencies application.
\end{abstract}

Keywords: Flexible device, Ink-jet printing, Printed electronics, Transmission line

\section{Background}

In recent years, printed electronics has received fast growing interest as alternative manufacturing method, because conventional processes, which are required etching and deposition processes, generate large amount of toxic chemical wastes during lift-off and electroplating process [1-4]. Therefore, many researchers started to pay attention on printed electronics that the etching or deposition processes are not required. In addition, printed electronics technology has the striking advantages such as simple process, low-cost, reduction of waste and capability of various substrates, compared with conventional processes.

Printed electronics device is fabricated by several printing methods including the screen printing, gravure printing, and ink-jet printing [5-8]. First, screen printing was widely used from long ago because it had simple printing tools which included stencil and squeegee. However, screen printing is not suitable for fragile substrate due

\footnotetext{
*Correspondence: jungmukim@jbnu.ac.kr

${ }^{3}$ Division of Electronic Engineering, Chonbuk National University, Jeonju 561-756, South Korea

Full list of author information is available at the end of the article
}

to inevitable contact of stencil with substrate and is not inexpensive because it accompanies large amount of ink. Furthermore, this printing method has a lower resolution than other printing methods. In gravure printing, ink was directly transferred through engraved roll. For this reason, it easily applies to roll-to-roll (R2R) process. Gravure printing has benefit of higher throughput because this printing method have advantages such as printable large area and fast printing speed by continuous process. However, a developed R2R printing equipment can be used by ink of one-type and printed structure can be damaged by contact of substrate with roll. Ink-jet printing technology creates a flexible electronics without contact of nozzle with substrate, which produces single droplet contained metal nano-particle through nozzle. This printing method offers relatively lower throughput than other printing methods, because ink-jet printer restricts the number of nozzles and directly draws a circuits of electronics device. However, the resolution is relatively higher than other printing methods, and various substrates are broadly utilized in order to use benefits of ink-jet printing technology. In addition, ink-jet printing provides distinguished advantage such as drop-ondemand printing, no wastage of ink, various functional 
inks, and non-contact printing. For this reason, research on ink-jet printing technology is required. In particular, RF performance of printed structure has to be investigated in regard to type of substrate because flexible device is fabricated on flexible substrate rather than rigid substrate.

In this paper, we investigated the influence of the rigid and flexible substrate about ink-jet printed transmission lines. The transmission lines are fabricated using ink-jet printing technology on rigid substrate (FR-4) and flexible substrate (PI). The RF performance of transmission lines is simulated and measured in the range from $300 \mathrm{kHz}$ to $1 \mathrm{GHz}$. The measured RF performance agrees well with tendency of simulation results and has potential in radio frequency identification (RFID) and smart label applications by simple and low-cost inkjet printing.

\section{Fabrication}

\section{Experimental setup}

The ink, DGP 40LT-15C (Advanced Nano Product Co., Korea), was used to print transmission lines. The ink was composed of $30.50 \mathrm{wt} \%$ silver nanoparticles with average size of approximately $20 \mathrm{~nm}$. The specific resistance of the ink was $11-12 \mu \Omega \mathrm{cm}$, and the viscosity was $17.3 \mathrm{cPs}$ at $100 \mathrm{rpm}$. The printing system was composed of a printhead equipped with nozzles of diameter of $19 \mu \mathrm{m}, \mathrm{XY}$ stage motorized with a positioning accuracy of $\pm 2 \mu \mathrm{m}$, and alignment system. The ink was ejected using ink-jet printer, Dimatrix DMP-2831 (Fujifilm Dimatix Inc., USA), with drop-on-demand from the substrate above $2 \mathrm{~mm}$. The substrates were patterned by FluoroCarbon (FC) solution to make the structure with high aspect ratio. The FC solution was composed of FC-722 and FC-40 (3M Co., Korea), which the spin-coated surface by the solution was known to have higher hydrophobicity. We used FR4 and PI substrate with the thickness of $1.6 \mathrm{~mm}$ and $200 \mu \mathrm{m}$, respectively, as rigid and flexible substrates, respectively. The SubMiniature version A (SMA) connectors with gap of 0.8 and $1.6 \mathrm{~mm}$ were used to measure RF performance of transmission lines.

\section{Design and fabrication process}

Figure 1 shows the schematic diagram of the transmission line. As shown in Table 1, two transmission lines are designed according to substrate in order to investigate the RF performance on both rigid and flexible substrates, which employed FR-4 and PI substrates, respectively. The parameters $h, l, w, t$ are designed to match the $50 \Omega$ characteristic impedance of transmission line. In the rigid transmission line, height of FR-4 substrate with 4.4 of permittivity is $1.6 \mathrm{~mm}$ and the width of the signal line should be $3.3 \mathrm{~mm}$ to match $50 \Omega$ characteristic impedance. The length of transmission line is set to $100 \mathrm{~mm}$. It

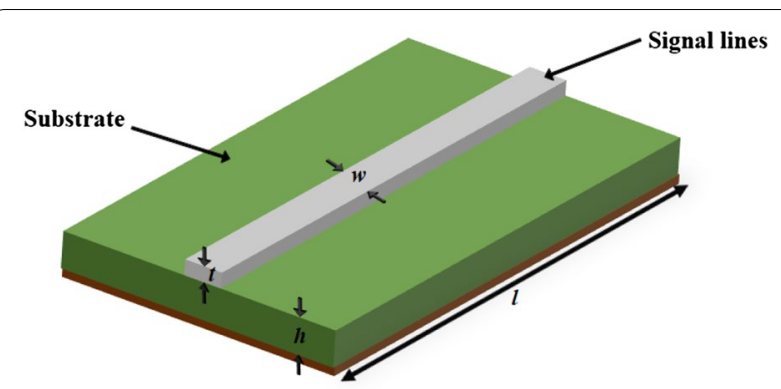

Fig. 1 Schematic diagram of transmission lines

Table 1 Design parameters of the transmission lines

\begin{tabular}{lll}
\hline & Transmission line \#1 & Transmission line \#2 \\
\hline Substrate & FR-4 & PI \\
Ground & Copper & Copper tape \\
Height of substrate $(h)$ & $1.6 \mathrm{~mm}$ & $0.2 \mathrm{~mm}$ \\
Length of substrate $(l)$ & $100 \mathrm{~mm}$ & $83 \mathrm{~mm}$ \\
Width of silver $(w)$ & $3.3 \mathrm{~mm}$ & $0.5 \mathrm{~mm}$ \\
Thickness of silver $(t)$ & $5 \mu \mathrm{m}$ & $3 \mu \mathrm{m}$ \\
\hline
\end{tabular}

is desirable to make the transmission line thicker than the skin depth to reduce the loss by skin effect because most of the RF current flows near the surface of signal line. The skin depth of silver is $2.8 \mu \mathrm{m}$ at $500 \mathrm{MHz}$, which reduces as the frequency increases. For this reason, thickness of signal line is designed to be $5 \mu \mathrm{m}$. In the flexible transmission line, the height and length of PI substrate with 3.1 of permittivity is 0.2 and $83 \mathrm{~mm}$, respectively. The width of transmission line is $0.5 \mathrm{~mm}$ to match $50 \Omega$ characteristic impedance, at the PI substrate with thickness of $0.2 \mathrm{~mm}$. The thickness of signal line is designed to be $3 \mu \mathrm{m}$ to reduce the loss by skin depth of silver.

The fabrication process of rigid transmission line is shown in Fig. 2A. First, FR-4 substrate deposited copper layers was prepared for rigid substrate. AZ 4330 photoresist was patterned on FR-4 substrate using the photolithography process. The patterns had the same as the dimensions of printed structure. The FC solution was spin-coated for $60 \mathrm{~s}$ at $1500 \mathrm{rpm}$ and baked at $110^{\circ} \mathrm{C}$ for 10 min. on a hot plate. The FC solution made the region where the ink didn't be easily printed on surface of substrate by its hydrophobic, whereas the non-coated region was hydrophilic rather than the coated region. The silver nanoparticle ink was then printed by using ink-jet printer with velocity of $3.5 \mathrm{~mm} / \mathrm{s}$ on non-coated region. The single droplet with volume of about $8 \mathrm{pL}$ was repeatedly ejected to realize the transmission line, which had thickness of $5 \mu \mathrm{m}$. The printed transmission line was sintered in a convection oven, which a temperature was set 


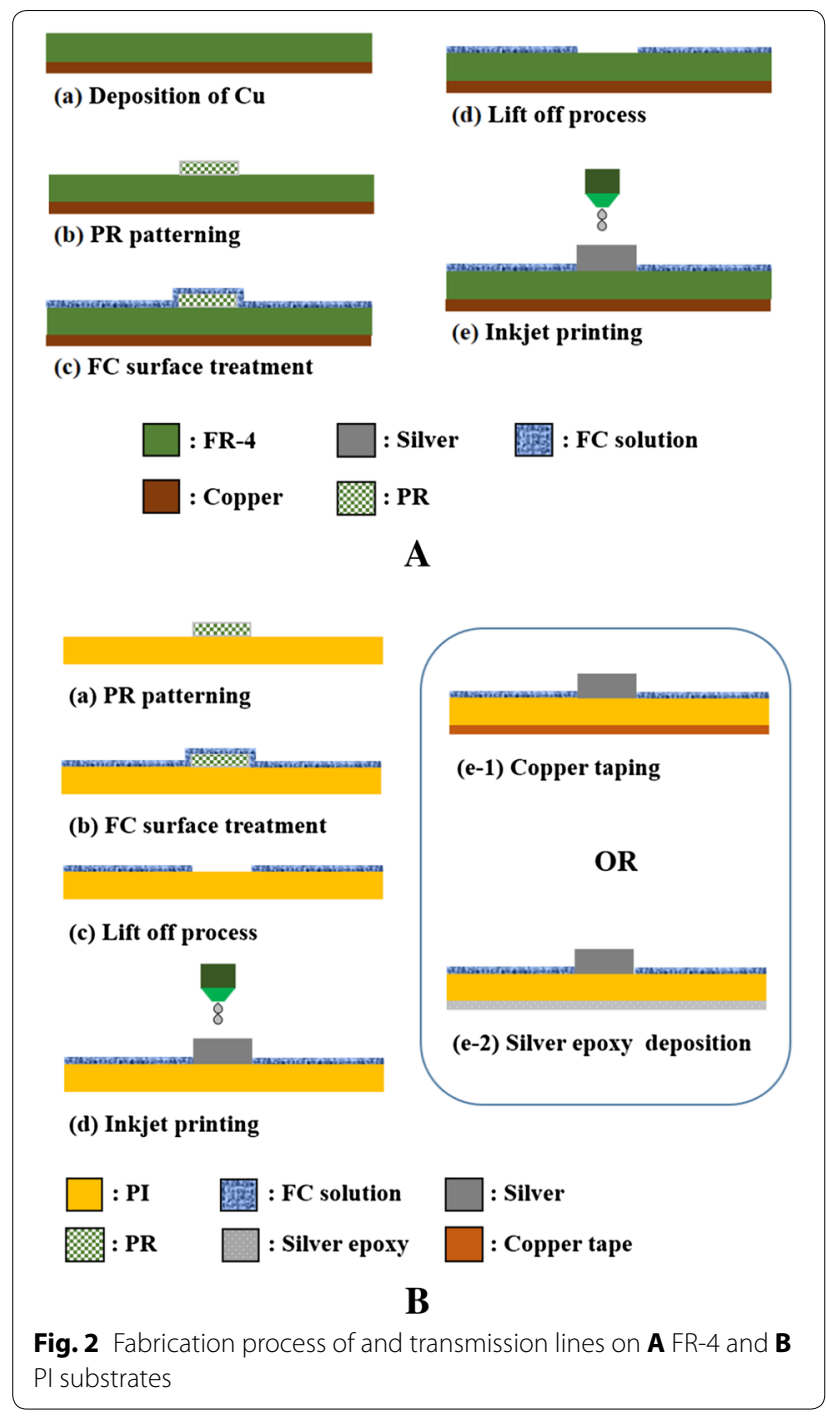

to $250{ }^{\circ} \mathrm{C}$ during $30 \mathrm{~min}$ to evaporate the TGME solvent of the printed structure. The silver nanoparticles were crystalized during the sintering process, which resulted in the higher adhesion force between the printed structure and substrate, and higher conductivity of printed transmission line. As shown in Fig. 2B, fabrication processes of flexible transmission lines are very similar with the rigid transmission line except for their ground and substrate. The fabrication of flexible transmission line was progressed through same process from patterning of photoresist process to sintering process. However, a commercially available copper tape, (Ducksunghitech Co., Korea), was used for ground layer of flexible substrate, because the vacuum deposition of copper was not simple on PI substrate.

\section{Results and discussion}

After the sintering process, the sheet resistance was measured using 4-point probe (MS TECH Co., Korea), which is $0.15 \Omega / \square$. It is expected that the RF performance of the printed transmission line is inferior to that fabricated by conventional processes, because of a lower conductivity of printed transmission line. Figure 3a shows the fabricated transmission line using inkjet printing on FR-4 substrate. Figure $3 \mathrm{~b}$ shows that the signal line printed using ink-jet printer and the grounds fabricated using copper tape. In Fig. 3b, c, a bulk cooper sheet with thickness of $600 \mu \mathrm{m}$ is also attached at both the edges of transmission lines to measure the RF performance of the transmission line on PI substrate.

To investigate the RF performance of the transmission lines on the rigid and flexible substrate, the RF performance was measured and compared. The frequency range was set to the low frequencies because the ink-jet printed structure has a low conductivity. RF simulation was performed at frequency ranging from $300 \mathrm{kHz}$ to $1 \mathrm{GHz}$, using Ansoft HFSS ${ }^{\mathrm{TM}}$ (Ansys lnc., USA). The conductivity of $3.33 \times 10^{7} \mathrm{~S} / \mathrm{m}$ was used for simulation, which was same dimension as fabricated transmission lines. The relative dielectric constant of FR-4 and PI substrates were also used 4.4 and 3.1, respectively. As shown in Fig. 4a, the transmission loss of the transmission line on FR-4 and PI substrate is simulated to be 0.038 and $0.043 \mathrm{~dB} / \mathrm{cm}$ at $1 \mathrm{GHz}$, respectively. The return loss for FR-4 and PI substrates is mostly better than $18 \mathrm{~dB}$, as shown in Fig. 4b. The transmission loss and return loss of the transmission lines were measured from $300 \mathrm{kHz}$ to $1 \mathrm{GHz}$, using vector network analyzer, E5061B (Agilent Technologies Co., USA), after Short-Open-LoadThrough (SOLT) calibration. The transmission loss of the transmission line on FR-4 and PI substrate is measured to be 0.12 and $0.22 \mathrm{~dB} / \mathrm{cm}$ at $1 \mathrm{GHz}$, respectively. The return loss is mostly better than 16 and $12 \mathrm{~dB}$, respectively, for FR-4 and PI substrates, respectively, at measured frequency. The measured RF performance shows moderate levels, while they are inferior to the simulation results. The nanoholes and cracks of the transmission line can be the cause of the high transmission loss because the TGME solvent was vaporized in ink-jet printed structure during the sintering process. The nanoholes and cracks are known that a higher roughness of CPW surface increase the RF current flow. In addition, the silver paste with conductivity of $1.0 \times 10^{6} \mathrm{~S} / \mathrm{m}$ is used as material to connect transmission line to SMA connector. An impedance mismatch can be another reason for the difference between the simulation and the measurement results, which take place in connection 


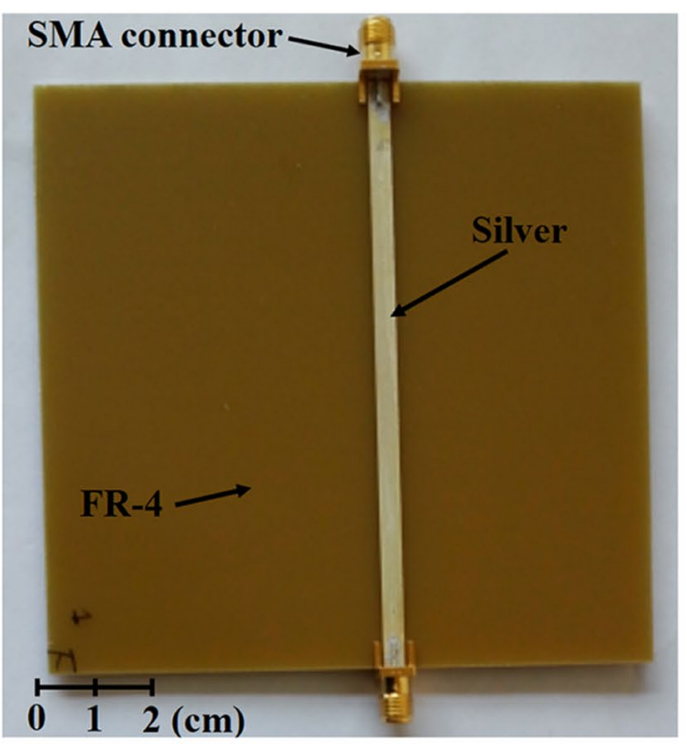

$\mathbf{a}$

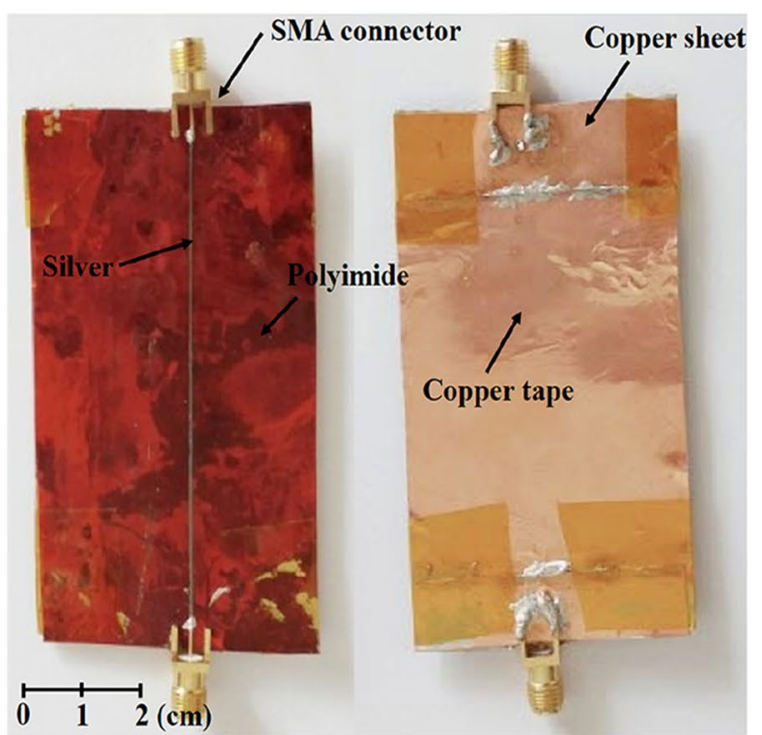

b

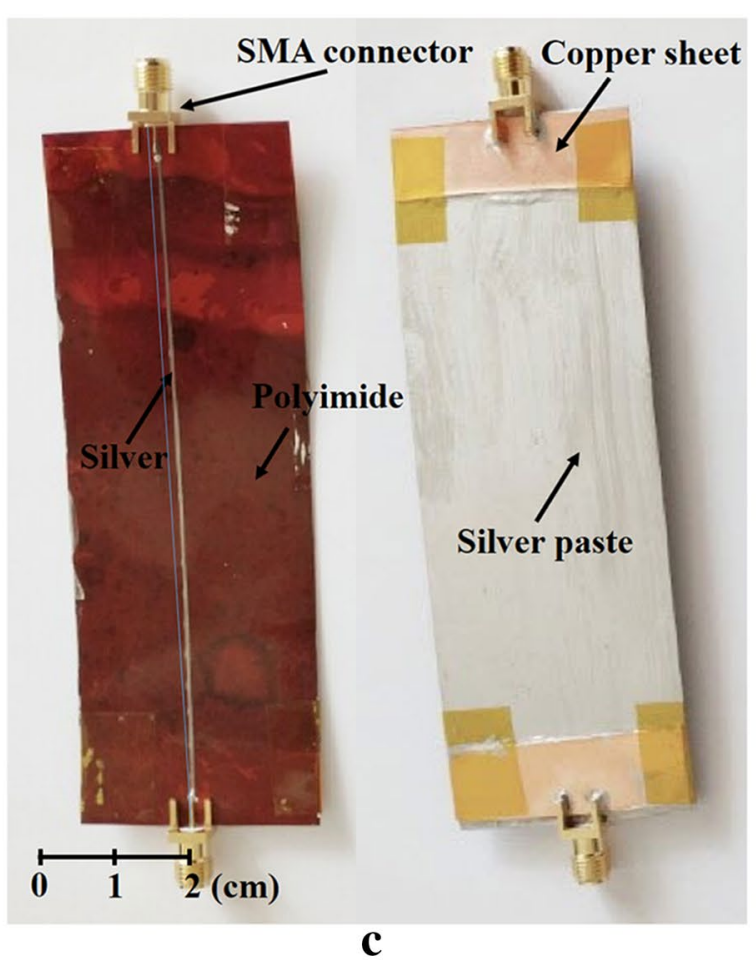

Fig. 3 Photograph of fabricated transmission lines. a Transmission line on FR-4 substrate, $\mathbf{b}$ transmission line on PI substrate with copper tape, c transmission line on PI substrate with silver paste

between SMA connector and edge of transmission line. As shown in Fig. 4a, the transmission loss of transmission line on flexible substrate shows the almost similar performance with the transmission line on rigid substrate. The slight difference between the transmission loss on FR-4 and PI substrates is regarded as a loss by the thin printed structure of $3 \mu \mathrm{m}$ thickness and the adhesion between the copper tape and PI substrate. Because the thickness of printed structure is required over $3 \mu \mathrm{m}$ by skin depth of silver under $500 \mathrm{MHz}$, the transmission line on PI substrate have higher the transmission loss under $500 \mathrm{MHz}$. Additionally, copper tape wasn't perfectly adhered, and a 


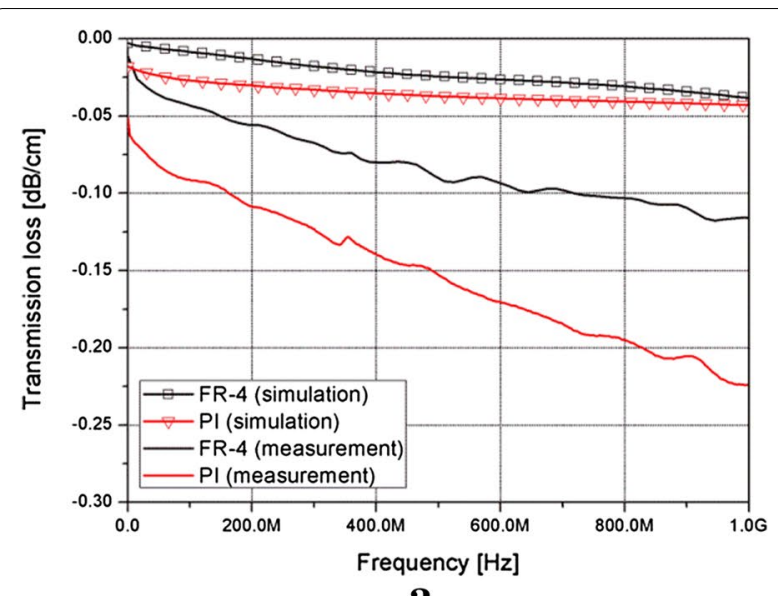

a
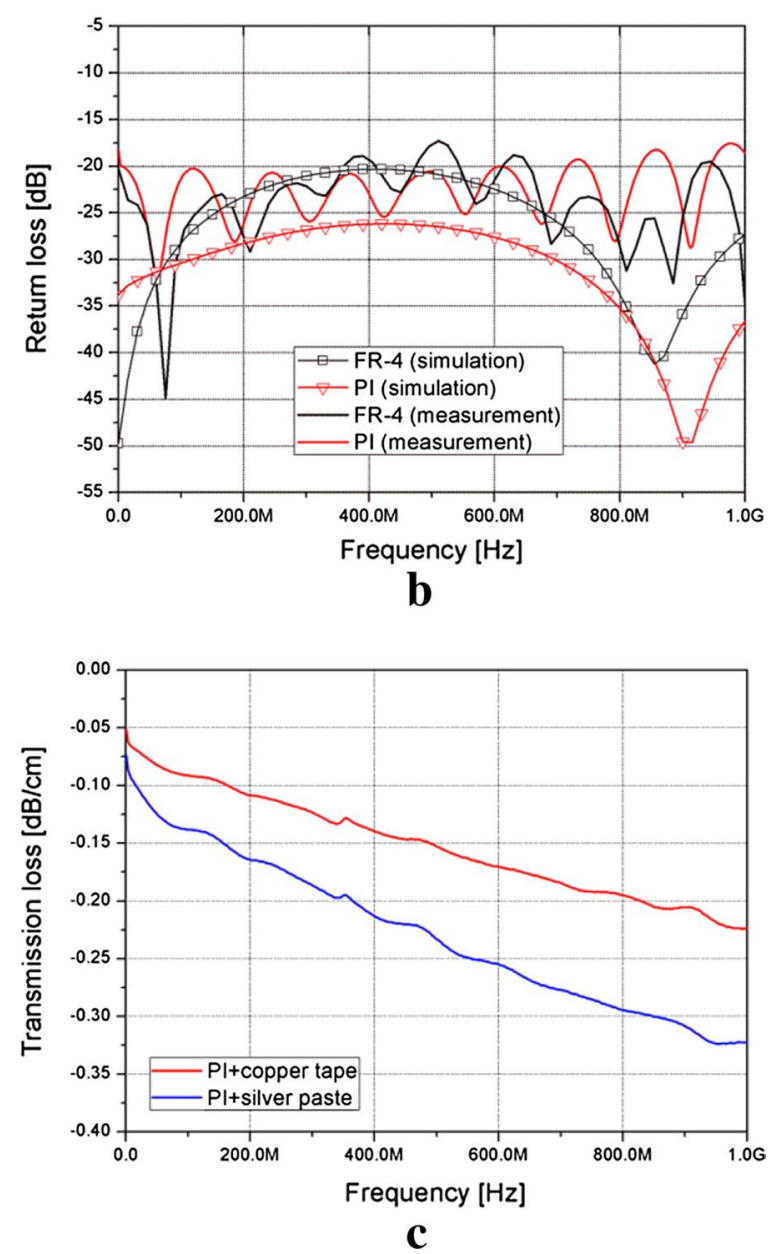

Fig. 4 RF performance of FR-4 and PI substrates, a the transmission loss of transmission lines, $\mathbf{b}$ the return loss of transmission line and c the measured results of transmission lines on PI substrate with ground layer of copper tape and silver paste small air bubbles between copper tape and PI substrate generate a parasitic capacitance in the process attaching copper tape. The parasitic capacitance attributes the higher transmission loss to the mismatching characteristic impedance of the transmission line on PI substrate. Nevertheless, the transmission lines can be used in wide range of electronic devices.

In order to resolve the parasitic capacitance by the air bubbles between the PI substrate and copper tape, the transmission line with the ground layer of the silver paste is additionally fabricated, as shown in Fig. 3c. The dimension and material property of the transmission line is same to transmission line \#2 except for the length of transmission line, as shown in Table 1. The length is $91 \mathrm{~mm}$, which is transmission line with the ground layer of silver paste. Although the transmission line for the ground layer of copper tape and silver paste were fabricated with different length, however the loss was measured and calculated per unit length to compare the proposed transmission lines. Figure 4c shows the RF performance of transmission lines with ground layer using the copper tape and silver paste. The transmission loss is measured to be 0.22 and $0.32 \mathrm{~dB} / \mathrm{cm}$ at $1 \mathrm{GHz}$, respectively. Although the ground layer of silver paste is used for improvement of RF performance, transmission loss is increase than previous transmission line because the silver paste has the lower conductivity than copper tape. Consequently, the results show that the conductivity of ground is important factor at RF performance of transmission line.

In conclusion, a transmission lines have almost fabricated using a vacuum deposition, electroplating and electroless plating $[9,10]$, however these transmission lines are difficult to be fabricated as the application in regard to flexible electronics device in the future [11]. Thus, we fabricated the transmission line on FR- 4 and PI substrates and investigated their RF performance in order to know an influence of substrate in regard to transmission lines. The RF performance of fabricated transmission lines on FR-4 and PI substrates are compared, as shown in Table 2. The RF performance shows a negligible difference according to the types of substrate. For this reason, inkjet printed technology can be utilized on the PI substrate almost any without degradation of RF performance.

\section{Conclusion}

In this study, we fabricated the ink-jet printed transmission line on FR-4 and PI substrates and measured the RF performance of transmission line. The transmission 
Table 2 Comparison of RF characteristic of fabricated transmission lines

\begin{tabular}{|c|c|c|c|c|c|}
\hline & Simulation & & Measuremen & & \\
\hline Substrate & FR-4 & PI & FR-4 & PI & $\mathrm{PI}$ \\
\hline Ground & Copper & Copper & Copper & Copper tape & Silver paste \\
\hline Transmission loss (dB/cm) & 0.038@1 GHz & 0.043@1 GHz & $0.12 @ 1 \mathrm{GHz}$ & 0.22@1 GHz & 0.32@1 GHz \\
\hline Return loss (dB) & $>18$ & $>18$ & $>16$ & $>12$ & $>12$ \\
\hline
\end{tabular}

loss of transmission line on FR-4 and PI substrate is 0.12 and $0.22 \mathrm{~dB} / \mathrm{cm}$ at $1 \mathrm{GHz}$, respectively. The transmission losses have a negligible difference even if additional transmission loss occurs by the ground layer at the transmission line on PI substrate. Therefore, the fabricated transmission lines show the possibility that flexible device can be used as a transmission line in UHF applications. Furthermore, we believe that the transmission line is alternative for conventional transmission line by low-cost, simple fabrication process in electronics device required performance of moderate level at low frequencies.

\section{Authors' contributions}

JMK conceived the idea and supervised the project. JMK, SHL, KYS and SMS discussed the design and the fabrication process of the ink-jet printed transmission line. SMS, YSL and HLK performed the measurement of RF performance and analysis of the results. JMK and SMS drafted the manuscript. All authors read and approved the final manuscript.

\section{Author details}

${ }^{1}$ Department of Electronic and Information Engineering, Chonbuk National University, Jeonju 561-756, South Korea. ${ }^{2}$ Korea Institute of Industrial Technology, Ansan 15588, South Korea. ${ }^{3}$ Division of Electronic Engineering, Chonbuk National University, Jeonju 561-756, South Korea.

\section{Acknowledgements}

This work was supported by research funds from the Korea Institute of Industrial Technology (KITECH).

\section{Competing interests}

The authors declare that they have no competing interests.

Received: 9 June 2016 Accepted: 25 August 2016

Published online: 10 September 2016

\section{References}

1. Fuller SB, Wilhelm EJ, Jacobson JM (2002) Ink-jet printed nanoparticle microelectromechanical systems. J Microelectromech Syst 11:54-60

2. Chung $\mathrm{WH}$, Hwang $\mathrm{HJ}$, Lee $\mathrm{SH}$, Kim HS (2013) In situ monitoring of a flash light sintering process using silver nano-ink for producing flexible electronics. Nanotechnology 24:035202-035209

3. Chang J, Ge T, Sanchez-Sinencio E (2012) Challenges of printed electronics on flexible substrates. In: 2012 IEEE 55th international midwest symposium on circuits and systems, pp 582-585

4. Subramanian V, Frechet JMJ, Chang PC, Huang DC (2005) Progress toward development of all-printed RFID tags: materials, processes, and devices. Proc IEEE 93:1330-1338

5. Szczech JB, Megaridis CM, Gamota DR, Zhang J (2002) Fine-line conductor manufacturing using drop-on demand PZT printing technology. IEEE Trans Electron Packag Manuf 25:26-33

6. Sridhar A, BlaudeckT, Baumann R (2011) Inkjet printing as a key enabling technology for printed electronics. Mater Matters 6:12-15

7. Lupo D, Clemens W, Breitung S, Hecker K (2013) Applications of organic and printed electronics. Springer US, New York City

8. Hrehorova E, Rebros M, Pekarovicova A, Bazuin B, Ranganathan A, Garner S, Merz G, Tosch J, Boudreau R (2011) Gravure printing of conductive inks on glass substrates for applications in printed electronics. J Disp Technol 7:318-324

9. Chamarti A, Varahramyan K (2006) Transmission delay line based ID generation circuit for RFID applications. IEEE Microw Wirel Compon Lett 16:588-590

10. Tuckerman DB, Hamilton MC, Reilly DJ, Bai R, Hernandez GA, Hornibrook JM, Sellers JA, Ellis CD (2016) Flexible superconducting Nb transmission lines on thin film polyimide for quantum computing applications. Supercond Sci Technol 29:084007-0840018

11. Wong WS, Salleo A (2009) Flexible electronics. Springer US, New York City

\section{Submit your manuscript to a SpringerOpen ${ }^{\circ}$ journal and benefit from:}

- Convenient online submission

- Rigorous peer review

- Immediate publication on acceptance

- Open access: articles freely available online

- High visibility within the field

- Retaining the copyright to your article

Submit your next manuscript at springeropen.com 